\title{
Erratum: Finite-temperature phase transition to a Kitaev spin liquid phase on a hyperoctagon lattice: A large-scale quantum Monte Carlo study [Phys. Rev. B 96, 125124 (2017)]
}

\author{
Petr A. Mishchenko, Yasuyuki Kato, and Yukitoshi Motome
}

(Received 30 January 2018; published 16 February 2018)

DOI: 10.1103/PhysRevB.97.079905

In our paper, there is an error in the values of the flux gap $\Delta$ in Table I. This error was corrected in the table presented below. Correspondingly, the sentence on p. 5 should read "The flux gap is estimated as $\Delta=0.030$ (3) for the hyperoctagon lattice and $\Delta=0.043$ (3) for the hyperhoneycomb lattice [1].” The error has no effect on our conclusions.

TABLE I. Comparison between the critical temperature $T_{c}$ obtained by the quantum Monte Carlo simulations and the magnitude of the flux gap $\Delta$ estimated at $T=0$ for the hyperhoneycomb and hyperoctagon cases. $T_{c}$ for the hyperhoneycomb system is taken from Ref. [2], whereas $\Delta$ is taken from Ref. [1].

\begin{tabular}{lcc}
\hline \hline Lattice & Critical temperature $T_{c}$ & Flux gap $\Delta$ \\
\hline Hyperoctagon & $0.00405(10)$ & $0.030(3)$ \\
Hyperhoneycomb & $0.00519(9)$ & $0.043(3)$ \\
\hline \hline
\end{tabular}

[1] K. O’Brien, M. Hermanns, and S. Trebst, Classification of gapless $Z_{2}$ spin liquids in three-dimensional Kitaev models, Phys. Rev. B 93, 085101 (2016).

[2] J. Nasu, M. Udagawa, and Y. Motome, Vaporization of Kitaev Spin Liquids, Phys. Rev. Lett. 113, 197205 (2014). 